\title{
Dividing attention across feature dimensions in statistical processing of perceptual groups
}

\author{
Tatiana Aloi Emmanouil and Anne Treisman \\ Princeton University, Princeton, New Jersey
}

\begin{abstract}
Statistical processing has been shown in the perception of several visual dimensions, including size, speed, direction of motion, and orientation. Chong and Treisman (2005) found no decrement when people simultaneously averaged two sets on a single dimension, size. What happens when attention is divided between different dimensions? In two experiments, we investigated judgments of mean size and speed, either within the same objects or in two separate sets. In a third, we examined judgments of mean size and orientation in two different sets. All three experiments suggest a decrement in performance when attention is shared between two dimensions, especially when they are carried by two different sets of objects.
\end{abstract}

Everywhere in the natural world, we see sets of objects with similar properties. The sizes of pebbles on a beach, the speed of cars on the highway, the orientations of branches blown by the wind provide a rich tapestry of sensory detail. However, specifying the size, color, form, and movement of each individual element would quickly overload the limited capacity of attention. In many cases, the relevant factors are the average properties of groups and the range of variation, rather than the detailed properties of each individual object. Does the visual system economize by encoding average properties?

Our ability to perceive statistical properties accurately has been demonstrated in a number of basic feature dimensions, such as size (Ariely, 2001; Chong \& Treisman, 2003), velocity and direction of motion (Atchley \& Andersen, 1995; Watamaniuk \& Duchon, 1992; Williams \& Sekuler, 1984), and orientation (Dakin \& Watt, 1997; Parkes, Lund, Angelucci, Solomon, \& Morgan, 2001). In the study by Ariely, participants viewed sets of circles of various sizes and judged whether a subsequently presented probe was larger or smaller than the mean size of the set. The results suggested that judgments of average size are quite accurate (diameter thresholds below 12\%) across a range of set sizes. Watamaniuk and Duchon (1992) asked participants to decide which of two cinematograms, in which dots moved with many different speeds, was faster. They found that the participants' judgments were based on the mean speed of all the dots and were independent of the mode and the variance. The ability to average orientations has been shown in the perception of textures (Dakin \& Watt, 1997) and of crowded Gabor displays (Parkes et al., 2001).

In contrast with the sharp capacity limits of focused attention, Treisman (2006) proposed that there may be a different mode of processing that occurs with distributed attention, yielding information about the gist and global organization of a scene and about the statistical properties of sets of similar objects within the scene. Capacity limits in this mode may be different from those found in the perception of individuated objects. For example, Chong and Treisman (2003) showed that average size judgments are almost as accurate with exposures as short as $50 \mathrm{msec}$ as they are with exposures of $1 \mathrm{sec}$, and as accurate in comparisons of the mean size of 12 heterogeneous circles as in comparing the size of 2 individual circles. Chong and Treisman (2005) also found that mean sizes can be computed for two different sets of circles simultaneously. They presented red and green circles in intermixed locations, distinguished only by the color of their outlines, and asked participants to judge the mean size of either the green or the red set. The critical factor was whether the cue indicating the set to be averaged was given before or after the participants viewed the display. When the cue was given before the display (precue), the participants could accurately perform the task by ignoring the irrelevant set. However, this strategy was not available when the cue was presented after the display (postcue), and the participants, unable to predict the type of cue, had to process both sets. Chong and Treisman (2005) found no significant decrement in the postcue condition. Moreover, performance was no better with one set presented alone than with two sets and a pre- or postcue, suggesting that averaging a single property in two different sets of stimuli occurs in parallel.

The surprising finding that people can compute more than one mean within the same dimension with no significant cost naturally leads to the question addressed in the present article: Can participants compute means on two different dimensions at once? In the experiments reported in this article, three dimensions were used in which statistical processing has previously been demonstrated: size, speed, and orientation. In Experiment 1, we presented

T. A. Emmanouil, temmanou@princeton.edu 
participants with sets of circles that varied in both size and speed of motion. On a given trial, the participants judged either the average size or the average speed of the circles. As in Chong and Treisman (2005), the cue, which in this case indicated which dimension to respond to, could appear either before (precue) or after (postcue) the display. In the precue condition, the participants could focus on the cued dimension, but in the postcue condition, successful performance required the processing of both dimensions. Any cost of dividing attention between dimensions would be reflected as a performance decrement in the postcue condition.

Divided attention experiments comparing single-object tasks with dual-object tasks have typically shown that it is easier to respond to different features of the same object than to different features of different objects (Duncan, 1984; Kahneman \& Henik, 1981; Treisman, 1969). Would the same be true of statistical processing across dimensions? That is, is it easier to average different features of the same set than to average different features of different sets? Whereas in Experiment 1 the participants averaged size and speed for the same set of objects, in Experiment 2 size and speed varied in two separate sets of objects. The displays of Experiment 2 presented stationary circles of different sizes, interspersed with Xs moving at different speeds. The task was to compute the average size of the circles or the average speed of the Xs, and the relevant dimension was again either precued or postcued. In Experiment 3 , we examined the cost of dividing attention between size and a new dimension, orientation, which was varied in a set of tilted bars.

\section{EXPERIMENT 1 Averaging Size and Speed Within One Set of Objects}

\section{Method}

Participants. Twenty-four participants (6 of them male, 18 female) took part in this experiment for course credit or for monetary compensation. The compensation rate was $\$ 10 / \mathrm{h}$. All the participants had normal or corrected-to-normal vision and gave written informed consent.

Task. The participants performed either the size or the speed task on different trials. In the size task, the participants indicated the side of the screen (right or left) with the largest mean size, whereas in the speed task they selected the side with the fastest mean speed. Importantly, the cue (the word "Size?" or "Speed?") informing the participants which task to perform appeared either before (precue) or after (postcue) the display of circles.

Stimuli. The stimuli were created in MATLAB 5 with the Psychophysics Toolbox (Brainard, 1997) and were presented using a Macintosh G4 computer on a Mitsubishi Diamond Pro 2070 SB monitor.

The stimuli were 16 circles of various sizes, moving upward at different speeds. The circles, 8 on the left side and 8 on the right side of the screen, as well as the fixation square $\left(0.36^{\circ}\right)$, were drawn in white on a gray background. The right and left sides of the screen were separated by a vertical black line, and no stimuli appeared within $1.2^{\circ}$ on either side of the line. All of the circles were centered within imaginary cells of $2.4^{\circ}$, and their positions were chosen so that during the course of their movement, they never overlapped. Motion

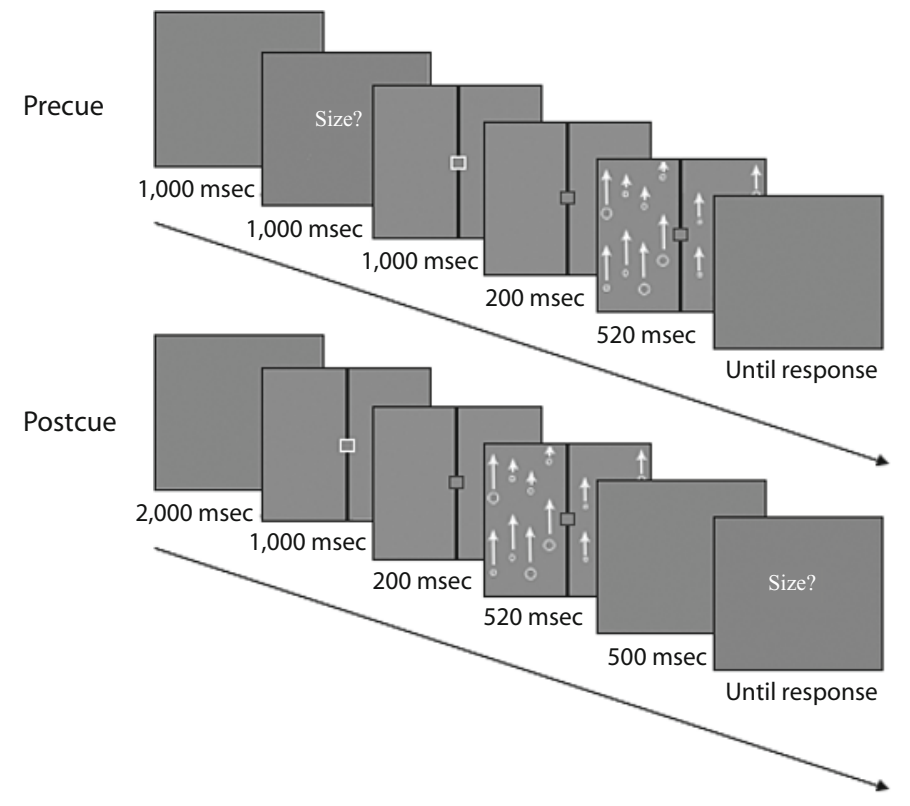

Figure 1. An example of a precue trial and a postcue trial for Experiment 1. The cue, fixation point, and dividing line have been enlarged, and the black area around the display has been omitted for the purposes of this figure. In the experiment, the fixation box turned red rather than dark gray. The figure shows the starting position of the circles, which then moved upward at different speeds (see the Method section). The circles that reached the top edge of the display were regenerated at the bottom. 
was produced by drawing the circles in a different position every two frames, with the refresh rate of the monitor set at $75 \mathrm{~Hz} .{ }^{1}$ The displacement of individual circles across frames was varied in order to achieve their desired velocity. Any circles that reached the top edge of the display were regenerated at the corresponding location at the bottom. The total display of circles was centered on the screen and subtended $21.7^{\circ} \times 19.7^{\circ}$, leaving the remaining screen area colored black.

Like Chong and Treisman (2005), we used two different methods to vary the mean size and speed of the circles on the two sides of the screen. On each trial, each method was randomly assigned to the right or the left side of the screen. According to the first method, the possible sizes and speeds of the circles were fixed, but their frequency varied. Circles were either small or large $\left(0.7^{\circ}\right.$ or $1.3^{\circ}$ diameter $)$ and either slow or fast $\left(5.4^{\circ}\right.$ or $13.5^{\circ}$ per second), resulting in four possible combinations (small-slow, small-fast, large-slow, and large-fast circles). ${ }^{2}$ There were four possible ratios of small to large and slow to fast circles ( 6 to 2,5 to 3,3 to 5 , and 2 to 6 ), and the assignments of size and speed remained uncorrelated. The second method, on the opposite side of the screen, created displays in which the frequency of different circles was fixed to two of each (small-slow, small-fast, large-slow, and large-fast) but the sizes and speeds were varied. The values associated with small, large, slow, and fast were adjusted so as to achieve the desired mean size and speed difference between sides $(6 \%, 12 \%, 18 \%, 24 \%, 30 \%$, or $36 \%)$, with mean size and mean speed determined independently on each trial. Differences in mean size were defined on a perceptual scale (Teghtsoonian, 1965), whereas differences in mean speed were linear. On half of the trials, the range of sizes and speeds on the equal-frequencies side of the display was the same as that on the opposite side; on the rest of the trials, it was equal to half the range on the opposite side. The range was independently manipulated for size and for speed.

Design. The three independent variables of interest were task (size or speed), cue (pre- or postcue), and discriminability (the difference in mean between the two sides of the screen on the cued dimension: $6 \%, 12 \%, 18 \%, 24 \%, 30 \%$, or $36 \%$ ). On each trial, the participants performed one of two tasks, the size or the speed task. The two task conditions were randomly intermixed within blocks, whereas the two cuing conditions appeared in separate blocks. The participants performed an equal number of trials for each of the four task-cuing combinations (size-pre, size-post, speed-pre, and speed-post). The largest mean size was on the opposite side from the fastest mean speed on half of the trials, so that the difference in means between the two sides of the screen for one dimension was not informative of the difference in means for the other dimension.

The first 16 participants completed one session consisting of 8 experimental blocks ( 4 of each cuing condition, 48 trials per block). The order of the cuing conditions was counterbalanced across participants. The remaining 8 participants participated in two separate sessions. Of those participants, 4 completed a total of 16 blocks ( 8 of each cuing condition), and 4 completed 18 blocks ( 9 of each cuing condition). Again, the order of the cuing conditions was counterbalanced. The reason that a second session was added for a subset of the participants is that the thresholds obtained with probit analysis on the basis of one session were, in some cases, too noisy to be usable: some too high (more than twice the highest level of discriminability) and some too low (below zero). This was particularly the case in the postcue condition.

Procedure. All the participants completed one precue and one postcue practice block (10 trials per block). The participants who completed two sessions received practice in the first session only.

Schematic representations of a precue and a postcue trial are shown in Figure 1. In the precue condition, a trial began with the cue, appearing on the screen for $1,000 \mathrm{msec}$. Immediately afterward, the fixation point and vertical dividing line appeared on the screen, and after 1,000 msec, the fixation point turned red, to alert the participants to the upcoming display. The circles appeared $200 \mathrm{msec}$ afterward and remained on the screen for approximately $520 \mathrm{msec}$ (motion was produced by the successive presentation of 20 frames on every other refresh of the moni-

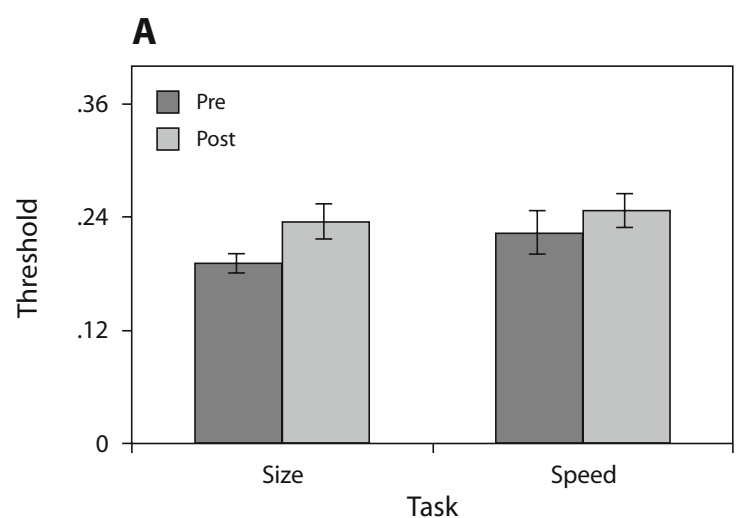

B

Size Task
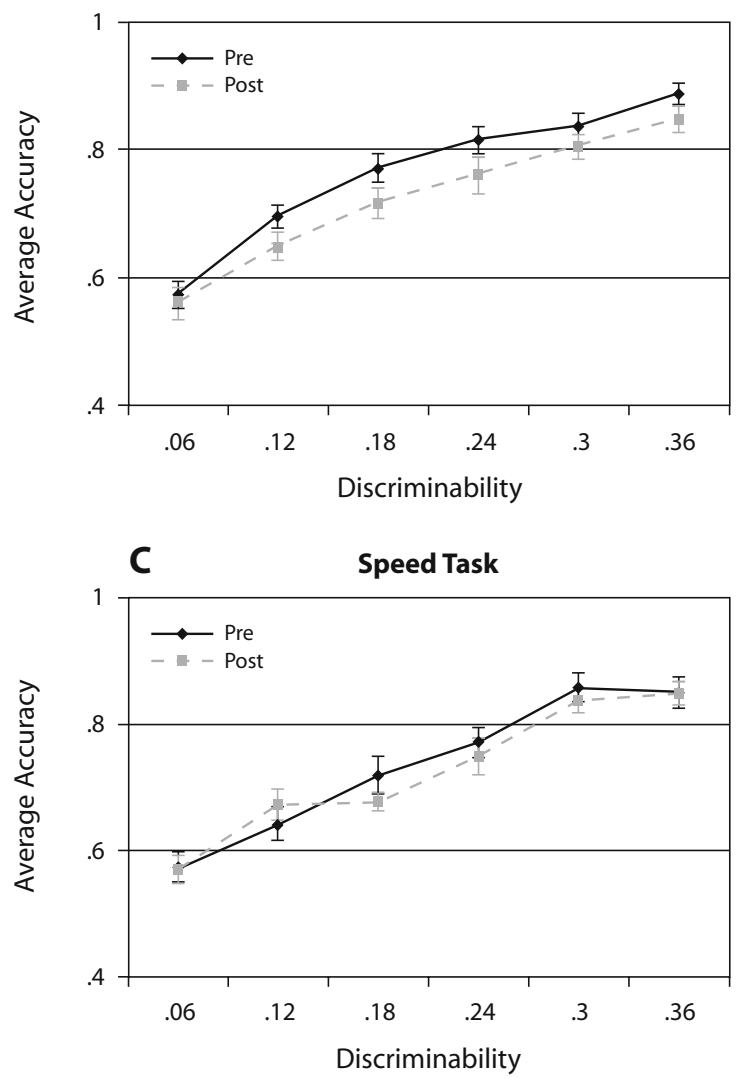

Figure 2. (A) Average 75\% accuracy thresholds for the precue (dark gray) and postcue (light gray) conditions in the size and speed tasks in Experiment 1. The later panels show average accuracy for the size (B) and the speed (C) tasks in the precue (dark solid line) and the postcue (light dashed line) conditions. Error bars indicate the standard errors of the means.

tor). The participants were given unlimited time to respond, and there was a 1,000-msec interval until the start of the next trial.

The postcue trials started with the appearance of the fixation point and the dividing line for $1,000 \mathrm{msec}$, followed by the fixation's turning red $(200 \mathrm{msec})$ and the circle display $(520 \mathrm{msec})$. The cue was 
presented $500 \mathrm{msec}$ after the offset of the circle display and stayed on the screen until the participant's response. In the postcue trials, the interval before the start of the next trial was $2,000 \mathrm{msec}$.

Participants pressed the " 1 " or " 2 " key on the keyboard number pad to indicate the left or right side of the screen, respectively. When their answer was incorrect, they heard a short, low-pitched tone.

Analysis. The data were analyzed using a repeated measures ANOVA and probit analysis. Probit analysis was used to obtain $75 \%$ accuracy thresholds for each participant. In the three experiments presented in this article, the participants with very high or very low thresholds (above twice the highest discriminability or below zero) were excluded from the analysis. Three participants were excluded from Experiment 1 on the basis of these criteria, all of whom had participated in one session only.

\section{Results and Discussion}

Figure 2 shows the $75 \%$ accuracy thresholds, as well as the average accuracy results, for the pre- and postcue conditions. A repeated measures ANOVA showed a main effect of cuing: Thresholds were significantly lower in the precue condition $[F(1,20)=6.77, p<.05]$. There was no main effect of task $[F(1,20)=1.5$, n.s. $]$ and no interaction between task and cue $[F(1,20)=0.95$, n.s.]. When the thresholds from the two tasks were analyzed separately, a significant precue advantage was found for the size task $[F(1,20)=7.72, p<.05]$, but not for the speed task $[F(1,20)=1.73$, n.s. $]$.

The raw accuracy data were analyzed with a repeated measures ANOVA on the effects of task, cue, and discriminability. Accuracy was significantly better in the precue than in the postcue condition $[F(1,20)=9.21, p<.01]$ but was similar for the two tasks $[F(1,20)=1.49$, n.s.]. The ANOVA also showed a main effect of discriminability $[F(5,100)=110.763, p<.01]$ and a marginally significant interaction between task and cue $[F(1,20)=3.34$, $p=.08]$, which suggests that the precue advantage was larger for the size task. There were no other significant two- or three-way interactions.

In this experiment, accuracy was found to be slightly higher in the precue than in the postcue condition: When participants could focus attention on the relevant task dimension, they performed better than when they divided attention between the two dimensions. The results suggest a cost in dividing attention between different dimensions in statistical processing. Note, however, that the precue advantage was small for the size task and did not reach significance for the speed task. Thus, although statistical processing is not optimal under conditions of divided attention, it still can be quite accurate.

The method we used involves comparing the means for the right and left sides of the screen. One may ask whether participants could perform the task by focusing attention on samples of just one or two items from each side (see Myczek \& Simons, 2008). For example, one plausible sampling strategy would be for participants to simply compare the largest or fastest items on each side. In this experiment, in order to reduce the correlation between the side that held the largest/fastest item and the side with the largest/fastest mean, we varied the range of sizes or speeds on the equalfrequencies side: On half the trials, the range was half that on the side with different frequencies of the two sizes/speeds.
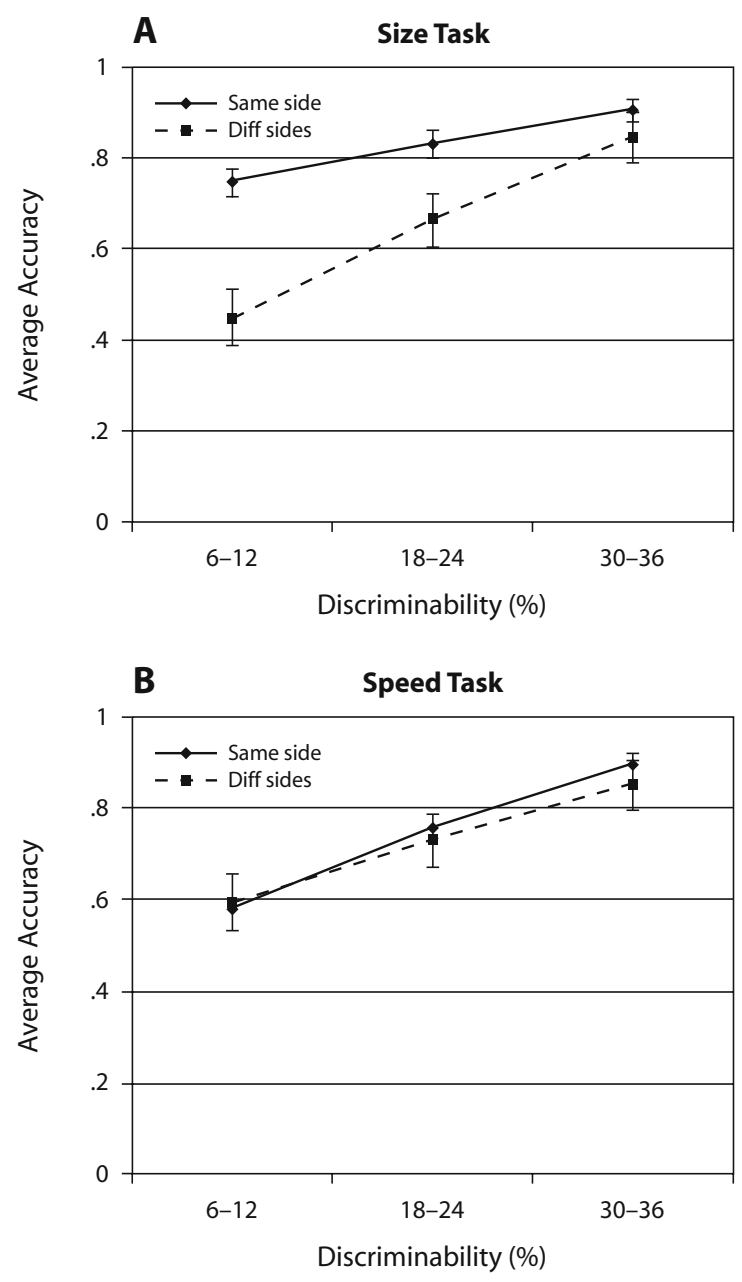

Figure 3. (A) Average accuracy for Experiment 1 size task trials on which the larger mean was on the same side as the larger item (solid line) and for trials on which the larger mean and larger item were on different sides (dashed line). (B) Average accuracy for trials in the speed task on which the faster item was on the same side as the faster mean (solid line) and for trials on which they were on different sides (dashed line). The data shown are precue trials on which the range in the equal-frequencies side is half the range on the opposite side. Error bars indicate standard errors of the means.

Figure 3 compares accuracy for the precued half-range trials on which the larger/faster mean and larger/faster individual item were on the same side and for trials on which they were on opposite sides. ${ }^{3}$ With speed judgments, there was no significant difference at all $[F(1,19)=0.16, p=.7]$, whereas with size, accuracy was higher when the larger average coincided with the larger individual item $[F(1,19)=$ $18.62, p<.01]$. However, note that performance on the trials on which the larger item and the larger mean were on opposite sides was well above chance. This is evidence against the use of the strategy, which would predict that performance on those trials should be close to zero (because judging based on the largest item would result in choos- 
ing the side with the smallest mean). Thus, even though the largest items may have influenced performance to some extent, the results indicate that the participants could not have responded solely on the basis of the comparison of the larger and faster individual items on each side.

So far, we have addressed the strategy of sampling the largest and fastest items. Is it possible that the participants used other sampling strategies in performing the task? Although we cannot eliminate this possibility, we believe that it is unlikely. In a series of experiments, Chong, Joo, Emmanouil, and Treisman (in press) provided evidence against the idea that participants perform statistical judgments by focusing attention on small samples of items (Myczek \& Simons, 2008). Chong et al. showed that even when sampling strategies are available, participants seem not to use them, supporting the idea that statistical averages are computed under a distributed, as opposed to a focused, deployment of attention.

Experiment 1 revealed a small cost of dividing attention to judge means on two different dimensions. This contrasts with the surprising finding by Chong and Treisman (2005) of parallel statistical processing of two sets of objects within a single dimension. In Experiment 2, we asked whether the cost we observed across dimensions would increase when each dimension was carried by a separate set of objects.

\section{EXPERIMENT 2 Averaging Size and Speed in Different Sets of Objects}

\section{Method}

Twenty-six participants (11 of them male, 15 female) gave informed consent and took part in this experiment for course credit or for pay. Sixteen participants completed one session (8 blocks), and the remaining 10 participated in two sessions ( 18 blocks, except for 1 participant who completed 16 blocks). Eight participants were excluded (see the Method section in Experiment 1 for exclusion criteria), of whom 6 had completed one session only. One two-session participant showed poor thresholds on the second session, and for this participant, only the data from the first session were used.

The methods in this experiment were very similar to those in Experiment 1. However, in Experiment 2, we used two sets of stimuli, circles and Xs, and varied each of them along only one dimension. The circles varied in size, and the Xs in speed of motion. Thus, the size and speed tasks were performed on different, spatially intermixed sets of objects. In the size task, the participants indicated the side of the screen on which the mean size of circles was largest, and in the speed task, they reported the side on which the speed of the Xs was greatest.

Eight stationary circles and eight moving Xs were presented on each side of the screen. The Xs subtended $0.54^{\circ}$ of visual angle. Occasionally in the course of motion, the Xs partly overlapped with one or more of the circles. The assignments of size and speed were the same as those in Experiment 1.

\section{Results and Discussion}

The results of Experiment 2 are presented in Figure 4. The repeated measures ANOVA on the $75 \%$ accuracy thresholds showed a main effect of cue $[F(1,17)=14.28, p<.01]$. As in Experiment 1, thresholds were lower in the pre- than in the postcue condition. There was no significant effect of task $[F(1,17)=2.19$, n.s. $]$ and no interaction between cue and task $[F(1,17)=0.39$, n.s.]. The two tasks were then analyzed separately, and the precue advantage was found to
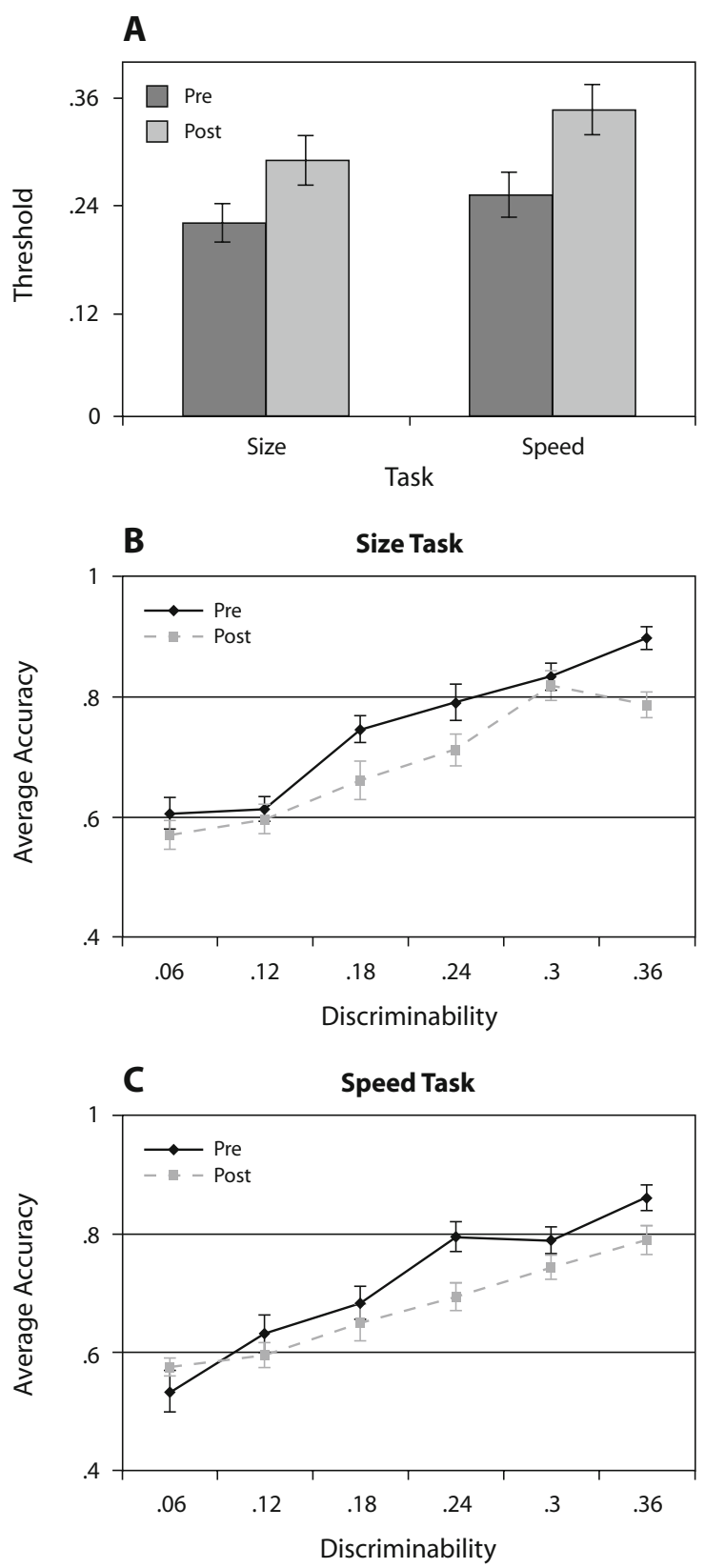

Figure 4. (A) Average thresholds and (B and C) average accuracy for size and speed tasks in Experiment 2. Error bars indicate standard errors of the means.

be significant for both the size $[F(1,17)=6.33, p<.05]$ and the speed $[F(1,17)=9.34, p<.01]$ tasks.

In order to see whether there was any increase in difficulty when the two dimensions were carried by different sets of objects, we compared the thresholds of Experiments 1 and 2 in a repeated measures ANOVA (task $\times$ cue), with experiment as a between-subjects variable. There was a main effect of experiment $[F(1,37)=6.82$, 
$p<.05]$, showing that performance was overall better in Experiment 1. Interestingly, the interaction between cue and experiment was very close to significant $[F(1,37)=$ $3.98, p=.054]$, suggesting that the precue advantage was larger in Experiment 2. There were no other significant two- or three-way interactions.

An overall ANOVA on the raw accuracy data for Experiment 2 also showed a significant precue advantage $[F(1,17)=19.03, p<.01]$ but no main effect of task $[F(1,17)=2.91$, n.s.]. There was a main effect of discriminability $[F(5,85)=89.94, p<.01]$ and an almost significant interaction between cue and discriminability $[F(5,85)=2.2, p=.053]$, suggesting that the precue effect varied between the different discriminability levels. This interaction could reflect a steeper psychometric function in the precue condition. We observed no other two- or three-way interactions.

This experiment corroborated the results of Experiment 1 , showing a clear precue advantage for both size and speed. The effect of the cue this time reached significance for both size and speed when the data were analyzed separately. The results again indicate that there is a cost in dividing attention between dimensions in statistical processing. The comparison of Experiments 1 and 2 suggests that the cost of sharing attention between dimensions is larger when the dimensions vary in two different sets of objects, as opposed to varying in a single set of objects, in the same way that responding to two individual features is more accurate when the two features belong to a single object rather than two separate objects (e.g., Duncan, 1984).

\section{EXPERIMENT 3 Averaging Size and Orientation Between Different Objects}

In Experiment 3, we investigated whether the cost of dividing attention would generalize to a new pair of dimensions, size and orientation. The two dimensions were varied in two separate sets of objects, as in Experiment 2. Size was varied in a set of stationary circles, and orientation in a set of rightward-tilted lines.

\section{Method}

Twenty-two participants ( 7 of them male, 15 female) were run in the experiment. All the participants gave informed consent and received course credit or monetary compensation for their participation. Fourteen participated in one session ( 8 blocks), and 8 participated in two sessions (18 blocks). Nine participants were excluded, according to the same criteria used in Experiments 1 and 2; of these, 5 had completed only one session.

The stimuli were stationary circles and oriented lines. The lines subtended $1^{\circ}$ of visual angle. They were tilted to the right of vertical, but by no more than $90^{\circ}$. The size task was to say on which side of the screen the circles had the larger mean size, and the orientation task was to indicate on which side of the screen the lines had the larger mean tilt from vertical. The cue was the word "Size?" or the word "Tilt?" The method for determining the size of the circles and the orientation of the lines on the two sides of the screen was the same as that in the previous experiments. The orientations used on the unequal-frequencies side (see Experiment 1, Method section) were $25^{\circ}$ and $50^{\circ}$ to the right of vertical. ${ }^{4}$ The sizes were the same as those used in Experiments 1 and 2.
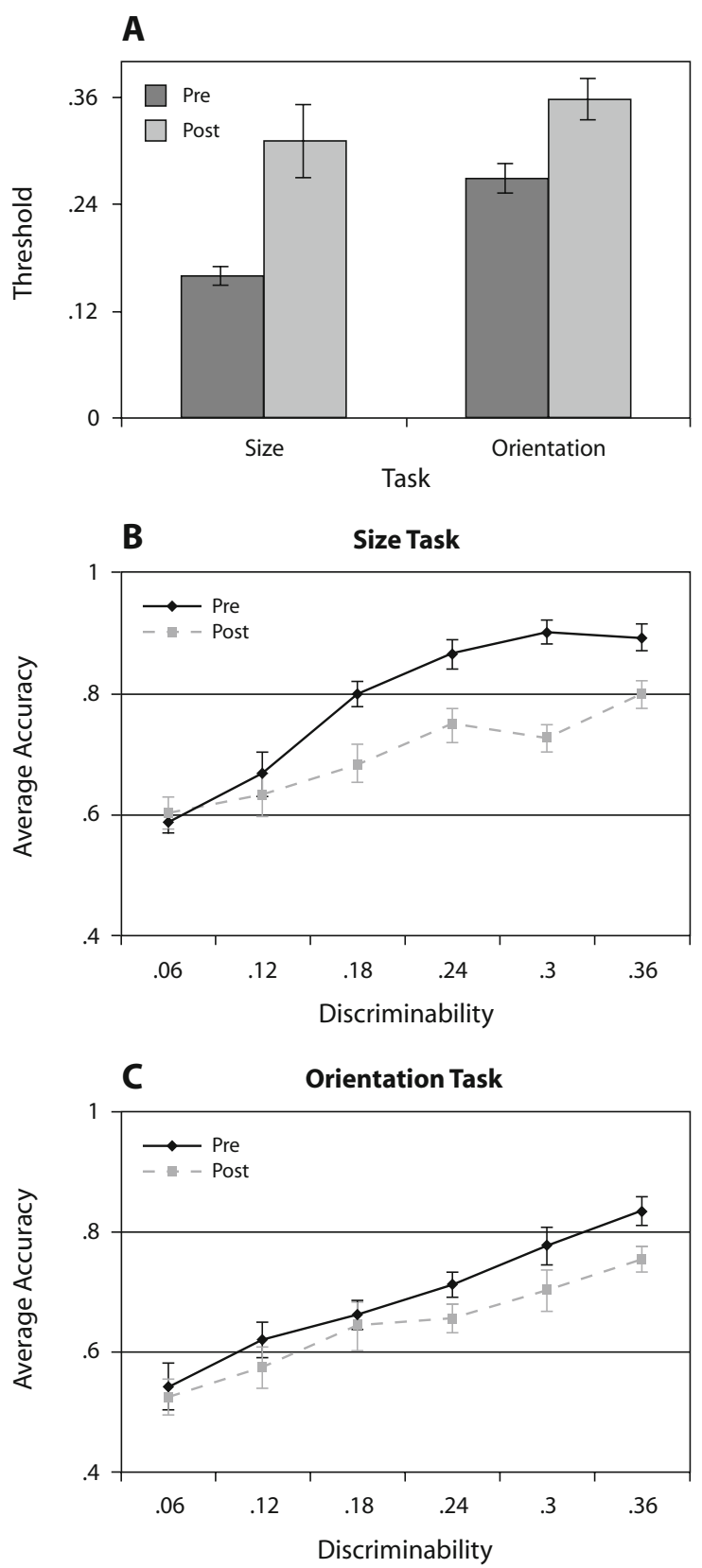

Figure 5. (A) Average thresholds and (B and C) average performance for the size and orientation tasks in Experiment 3. Error bars indicate standard errors of the means.

\section{Results and Discussion}

The results of Experiment 3 are presented in Figure 5. An overall ANOVA on the $75 \%$ accuracy thresholds showed a significant precue advantage $[F(1,12)=28.59$, $p<.01]$. There was also a main effect of task $[F(1,12)=$ $12.05, p<.05]$, showing that the participants were better in the size task, but there was no interaction between cue and task $[F(1,12)=1.32$, n.s.]. When the two tasks were 
analyzed separately, the precue effect was found to be significant in both the size $[F(1,12)=13.1, p<.01]$ and the orientation $[F(1,12)=10.56, p<.01]$ tasks.

The size judgments were compared across Experiment 2 and Experiment 3, to see whether there were any differences in dual-task interference from concurrently judging orientation and from concurrently judging speed. A repeated measures ANOVA on the size thresholds, with experiment as a between-subjects variable, showed no significant interaction between cue and experiment $[F(1,29)=2.75$, n.s. $]$ and no main effect of experiment $[F(1,29)=0.33$, n.s. $]$, suggesting that speed and orientation are equally easy or equally difficult to combine with size judgments.

The ANOVA on the accuracy data of Experiment 3 revealed significantly better accuracy in the size task than in the orientation task $[F(1,12)=44.61, p<.01]$ and in the pre- as opposed to the postcue condition $[F(1,12)=$ $33.54, p<.01]$. There was also a main effect of discriminability $[F(5,60)=41.4, p<.01]$ and an interaction between cue and discriminability $[F(5,60)=2.83, p<.05]$, suggesting that the effect of the cue varied among different discriminability levels. Again, the precue condition showed a steeper effect of discriminability.

The results of this experiment reveal a precue advantage in the statistical processing of size and orientation. Thus, this experiment shows a cost of dividing attention across a new pair of dimensions and suggests that the decrement observed in the previous two experiments is not specific to size and speed.

\section{GENERAL DISCUSSION}

In the three experiments described in this article, we examined whether the accuracy of statistical processing decreases when people divide attention between two different dimensions. In order to explore this possibility, we showed participants displays of elements with two different properties. On a given trial, they were asked to judge the average of one of the two properties. The relevant dimension was indicated by a cue, which appeared either before the display (precue) or after (postcue). The comparison between performance in the precue condition, in which the participants could perform well just by focusing on one dimension, and that in the postcue condition, in which successful performance required averaging on both dimensions, allowed us to examine the cost of dividing attention between dimensions in statistical processing.

In Experiment 1, we used circles varying in size and in speed of motion in order to assess performance when attention was either focused on one property or else divided between two properties of the same objects. A precue advantage was found, suggesting that there is a cost in dividing attention between size and speed in statistical processing. This precue advantage was confirmed in Experiment 2 , which tested statistical processing of size and speed as properties of two separate sets of objects (stationary circles and moving Xs). In fact, the precue advantage was larger in Experiment 2, suggesting that dividing attention between size and speed is harder when it means also dividing attention between different sets of objects, in agreement with findings in object perception research (e.g., Duncan, 1984). In Experiment 3, we examined statistical processing in another pair of dimensions, size and orientation, which, as in Experiment 2, varied in two separate sets of objects. The results again showed a precue advantage and suggest that the cost of switching dimensions in statistical processing generalizes to dimensions other than size and speed.

The comparison between the present results and those of Chong and Treisman (2005) is particularly relevant. Chong and Treisman (2005) found no decrement in performance when participants averaged two spatially intermixed sets along the same dimension, size. In the present experiments, we again asked participants to compute two means, this time on two separate dimensions. The results showed a decrement in performance, suggesting that there is a cost in the statistical processing of two means only when these are computed on separate dimensions, as opposed to a single one.

However, in comparing the results of the present study with those of Chong and Treisman (2005), a difference in method between the studies should be borne in mind. Our participants compared two simultaneously presented groups, one on the left and one on the right, to determine which had the larger, faster, or more tilted elements. Chong and Treisman's (2005) participants, on the other hand, viewed a single display of two sets of mixed elements and then indicated which of two subsequent probes matched the mean size of the cued set. Could the postcue decrement in the present experiments be explained by the fact that the participants were dividing attention not only between dimensions, but also between the two sides of the screen? Although possible, it is unlikely that comparing two sides that are simultaneously present would produce worse performance than judging a single display and then comparing the two means with a memory probe. In fact, the statistical thresholds obtained with the probe method were previously found to be higher than those produced by comparing two sides (Chong \& Treisman, 2003), presumably because of increased memory demands. Thus, in the present study, it is unlikely that the method of comparing two sides led to worse performance than did Chong and Treisman's (2005) method using a single display with a memory probe.

Of course, it is still the case that within the present experiment, the pre- and postcue conditions differed in their demands on memory. In the precue condition, participants had to store one piece of information, the side of the screen associated with a larger mean value in the relevant dimension (size, speed, or orientation). In the postcue condition, participants memorized the side with the largest mean value separately for each dimension. Could this difference in memory demands account for the observed accuracy cost in the postcue condition? We would argue that this explanation is unlikely, mainly on the basis of the results of Chong and Treisman (2005). They found no difference between the pre- and postcue conditions when participants averaged two sets of objects on the same dimension, suggesting that differences in memory load are 
not sufficient to produce an accuracy decrement in the postcue condition. Therefore, memory differences between the two conditions are unlikely to account for this decrement, which was found to be quite robust across the experiments reported in this article.

In the present study, we identified two sources of increased load in statistical processing: the number of relevant properties to be averaged and the number of sets of objects carrying those properties. The cost of dividing attention between different properties is demonstrated in the comparison of our results with those of Chong and Treisman (2005): Our results show that judging two means in two separate dimensions produces an accuracy cost, in contrast with Chong and Treisman's (2005) finding that processing two means in one dimension occurs in parallel. The cost of dividing attention between sets of objects is seen in the comparison of Experiments 1 and 2, which shows that averaging size and speed is somewhat harder when these vary in separate sets, as opposed to a single set, of objects.

Do these two factors-dividing attention between dimensions and dividing attention between objectssimilarly limit the processing of individual objects? The cost of dividing attention between separate objects, each varying on multiple dimensions, has been confirmed in several studies. First, there is a large literature exploring whether objects are scanned serially or in parallel, with evidence showing that simple discriminable features can be searched in parallel, whereas conjunctions of features (as well as features that are hard to discriminate) may require serial processing (Treisman \& Gelade, 1980; Treisman \& Gormican, 1988). In agreement with these findings, a number of studies have suggested that it is easier to judge multiple properties when they belong to a single object, as opposed to separate objects (e.g., Duncan, 1984). There has been less research on the other question-whether there is a limit to the number of dimensions that can be processed in parallel — and the results of existing studies are mixed. Duncan (1993) found no decrement in judging two different properties of two spatially separated objects, relative to judging the same property. Similarly, Lee, Koch, and Braun (1999) found no difference in the cost of dividing attention between a central and a peripheral object, whether the relevant dimension was the same or different.

Whereas these results provide evidence against a cost of switching between dimensions in the perception of separate objects, several studies by Müller and colleagues seem to support it. Müller, Heller, and Ziegler (1995) proposed a dimensional-weighting account, according to which the amount of attention allocated to different dimensions varies, on the basis of top-down expectations, and there is a cost in switching between them. Müller and colleagues have repeatedly demonstrated an intertrial cost when the relevant dimension defining a target switched across two successive trials, presumably reflecting the cost of switching between dimensions (Found \& Müller, 1996; Müller et al., 1995). According to dimensional weighting, the top-down weight each dimension receives influences the detection of targets defined on that dimension. Müller, Reimann, and Krummenacher (2003) indeed found that cuing the dimension in which the target was different from the distractors facilitated detection of the target.

Our results are consistent with dimensional weighting, showing that statistical processing is more accurate within a cued dimension. This effect could have two different explanations within the framework of dimensional weighting. It is possible that individual objects in our displays are processed in the cued dimension first and that this results in better perception and improved statistical processing within that dimension. Alternatively, statistical means could be independently computed within separate feature maps (much as the saliency of targets, relative to distractors, is computed in dimensional weighting), with the cued dimension receiving increased weight and better access to consciousness. In any case, our results are consistent with a cost of switching between dimensions, even though the level at which this cost arises is unclear.

The three experiments reported in this article suggest that simultaneously averaging two different dimensions results in a decrement in performance. Taken together with the results in Chong and Treisman (2005), our results suggest that attention to a feature dimension may be necessary for optimal statistical processing within that dimension, whereas a single dimension can be averaged in parallel across separate groups. Since statistical processing relies anyway on distributing attention over space across objects, it makes sense that it should be more efficient at processing multiple groups than at processing multiple dimensions, which is not a spatial load but, rather, a matter of applying different forms of analysis concurrently to incoming stimuli.

It is noteworthy, however, that performance was well above chance on both dimensions in all three experiments. There seems to be sufficient capacity to get at least rough estimates of the means on two dimensions, and if both vary in the same set of objects, the decrement is very small. The performance observed in these tasks, although clarifying some limits to attention in statistical processing of separate dimensions, nevertheless is consistent with our ability to form a rich global impression of natural scenes.

\section{AUTHOR NOTE}

This research was supported by NIH Grant 2004 2RO1 MH 05838304A1, by Grant 1000274 from the Israeli Binational Science Foundation, and by NIH Grant EY016975. Correspondence concerning this article may be addressed to T. A. Emmanouil, Psychology Department, Princeton University, Princeton, NJ 08540 (e-mail: temmanou@princeton.edu).

\section{REFERENCES}

ArIELY, D. (2001). Seeing sets: Representation by statistical properties. Psychological Science, 12, 157-162.

Atchley, P., \& Andersen, G. J. (1995). Discrimination of speed distributions: Sensitivity to statistical properties. Vision Research, 35, 3131-3144.

Brainard, D. H. (1997). The Psychophysics Toolbox. Spatial Vision, 10, 433-436.

Chong, S. C., Joo, S. J., Emmanouil, T. A., \& Treisman, A. (in press). Statistical processing: Not so implausible after all. Perception \& Psychophysics.

Chong, S. C., \& Treisman, A. (2003). Representation of statistical properties. Vision Research, 43, 393-404.

Chong, S. C., \& Treisman, A. (2005). Statistical processing: Com- 
puting the average size in perceptual groups. Vision Research, $\mathbf{4 5}$, 891-900.

DAKIN, S. C., \& WATT, R. J. (1997). The computation of orientation statistics from visual texture. Vision Research, 37, 3181-3192.

DunCan, J. (1984). Selective attention and the organization of visual information. Journal of Experimental Psychology: General, 113, 501-517.

DunCan, J. (1993). Similarity between concurrent visual discriminations: Dimensions and objects. Perception \& Psychophysics, 54, 425-430.

Found, A., \& MüLLER, H. J. (1996). Searching for unknown feature targets on more than one dimension: Investigating a "dimensionweighting" account. Perception \& Psychophysics, 58, 88-101.

Kahneman, D., \& HeniK, A. (1981). Perceptual organization and attention. In M. Kubovy \& J. R. Pomerantz (Eds.), Perceptual organization (pp. 181-211). Hillsdale, NJ: Erlbaum.

LeE, D. K., Koch, C., \& Braun, J. (1999). Attentional capacity is undifferentiated: Concurrent discrimination of form, color, and motion. Perception \& Psychophysics, 61, 1241-1255.

Müller, H. J., Heller, D., \& Ziegler, J. (1995). Visual search for singleton feature targets within and across feature dimensions. Perception \& Psychophysics, 57, 1-17.

Müller, H. J., Reimann, B., \& Krummenacher, J. (2003). Visual search for singleton feature targets across dimensions: Stimulusand expectancy-driven effects in dimensional weighting. Journal of Experimental Psychology: Human Perception \& Performance, 29, 1021-1035.

MyczeK, K., \& Simons, D. J. (2008). Better than average: Alternatives to statistical summary representations for rapid judgments of average size. Perception \& Psychophysics, 70, 772-788.

Parkes, L., Lund, J., Angelucci, A., Solomon, J. A., \& Morgan, M. (2001). Compulsory averaging of crowded orientation signals in human vision. Nature Neuroscience, 4, 739-744.

Teghtsoonian, M. (1965). The judgment of size. American Journal of Psychology, 78, 392-402.

Treisman, A. M. (1969). Strategies and models of selective attention. Psychological Review, 76, 282-299.

Treisman, A. [M.] (2006). How the deployment of attention determines what we see. Visual Cognition, 14, 411-443.
Treisman, A. M., \& Gelade, G. (1980). A feature integration theory of attention. Cognitive Psychology, 12, 97-136.

Treisman, A. [M.], \& Gormican, S. (1988). Feature analysis in early vision: Evidence from search asymmetries. Psychological Review, 95, 15-48.

VAN EYL, F. P. (1981). Velocity judgments of continuously moving and stroboscopically presented stimuli using magnitude and category scaling. Acta Psychologica, 48, 89-94.

Watamaniuk, S. N., \& Duchon, A. (1992). The human visual system averages speed information. Vision Research, 32, 931-941.

Williams, D. W., \& SeKuler, R. (1984). Coherent global motion percepts from stochastic local motions. Vision Research, 24, 55-62.

\section{NOTES}

1. A minority of trials (approximately $5 \%$ ) on which the computer unavoidably introduced an extra refresh was excluded from the analysis.

2. A multiplication factor of $1,1.1,1.2$, or 1.3 was used to independently scale both the sizes and the speeds of the circles on each trial, in order to prevent the participants from memorizing a limited set of stimuli. Each factor was used equally often, and the order was randomized. The scaling for size was performed on a power function with an exponent of .76, which has been shown to best predict perceived size for circles (Teghtsoonian, 1965), whereas the scaling for speed was linear (Van Eyl, 1981).

3. In the speed task, the average percentage of precue, half-range trials on which the faster items were dissociated from the faster mean was $23 \%$. In the size task, the average percentage of trials on which the larger items were on the opposite side from the larger mean was $26 \%$. We collapsed across pairs of adjacent discriminability differences, both in the figure and in the ANOVA. One participant was excluded because we had no trials with a dissociation in the speed task with a $30 \%-36 \%$ difference in discriminability.

4. A scale factor was applied as in Experiments 1 and 2, and the scaling of orientations was linear.

(Manuscript received October 24, 2007; revision accepted for publication February 18, 2008.) 